\title{
The mortality burden in patients with hip fractures and dementia
}

\author{
loannis loannidis ${ }^{1,2} \cdot$ Ahmad Mohammad Ismail $^{1,2}$ (1) $\cdot$ Maximilian Peter Forssten ${ }^{1,2}\left(\mathbb{C} \cdot\right.$ Rebecka Ahl $^{2,3} \cdot$ Yang Cao $^{4}$. \\ Tomas Borg $^{1,2} \cdot$ Shahin Mohseni ${ }^{2,5}$
}

Received: 13 November 2020 / Accepted: 5 February 2021 / Published online: 27 February 2021

(c) The Author(s) 2021

\begin{abstract}
Purpose Dementia is strongly associated with postoperative death in patients subjected to hip fracture surgery. Nevertheless, there is a distinct lack of research investigating the cause of postoperative mortality in patients with dementia. This study aims to investigate the distribution and the risk of cause-specific postoperative mortality in patients with dementia compared to the general hip fracture population.

Methods All adults who underwent emergency hip fracture surgery in Sweden between 1/1/2008 and 31/12/2017 were considered for inclusion. Pathological, conservatively managed fractures, and reoperations were excluded. The database was retrieved by cross-referencing the Swedish National Quality Registry for Hip Fracture patients with the Swedish National Board of Health and Welfare quality registers. A Poisson regression model was used to determine the association between dementia and all-cause as well as cause-specific 30-day postoperative mortality.

Results 134,915 cases met the inclusion criteria, of which $20 \%$ had dementia at the time of surgery. The adjusted risk of all-cause 30-day postoperative mortality was $67 \%$ higher in patients with dementia after hip fracture surgery compared to patients without dementia [adj. IRR (95\% CI): 1.67 (1.60-1.75), $p<0.001$ ]. The risk of cause-specific mortality was also higher in patients with dementia, with up to a sevenfold increase in the risk cerebrovascular mortality [adj. IRR (95\% CI): 7.43 (4.99-11.07), $p<0.001]$.

Conclusions Hip fracture patients with dementia have a higher risk of death in the first 30 days postoperatively, with a substantially higher risk of mortality due to cardiovascular, respiratory, and cerebrovascular events, compared to patients without dementia.
\end{abstract}

Keywords Hip fracture $\cdot$ Dementia $\cdot$ Postoperative mortality $\cdot$ Cause-specific mortality

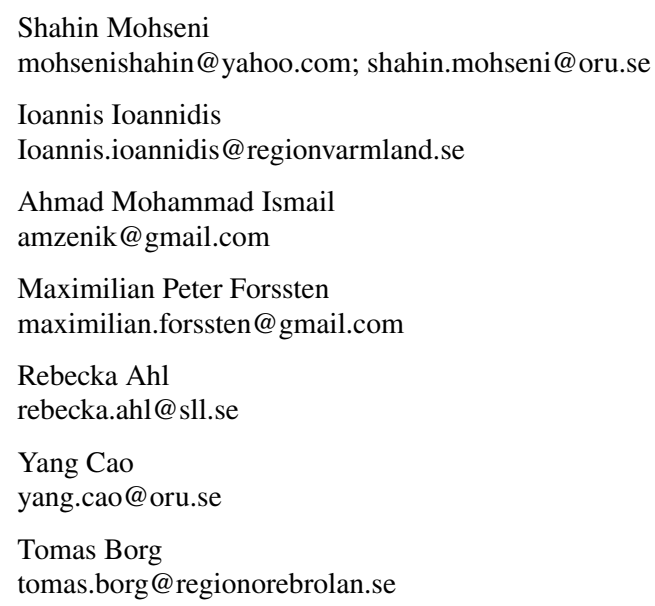

1 Department of Orthopedic Surgery, Orebro University Hospital, 70185 Orebro, Sweden

2 School of Medical Sciences, Orebro University, 70281 Orebro, Sweden

3 Division of Trauma \& Emergency Surgery, Department of Surgery, Karolinska University Hospital, 17176 Stockholm, Sweden

4 Clinical Epidemiology and Biostatistics, School of Medical Sciences, Orebro University, 70182 Orebro, Sweden

5 Division of Trauma and Emergency Surgery, Department of Surgery, Orebro University Hospital, 70185 Orebro, Sweden 


\section{Background}

The association between dementia and an increased risk of mortality after hip fracture operations is strongly backed by scientific evidence in the field of orthopedic surgery [1-4]. The volume of hip fractures is projected to continue to grow globally due to an aging population [5]. Currently, the cumulative lifetime risk of suffering a hip fracture after the age of 50 is $24 \%$ in women and $11 \%$ in men in Sweden [6]. In addition to other medical conditions which are associated with worse postoperative outcomes, up to $23 \%$ of hip fracture patients suffer from dementia [7, 8]. By 2050, the prevalence of dementia is projected to have doubled [9]. These numbers are consistent with those predicted worldwide [10].

There is a paucity in research that studies the cause of death in patients with dementia after hip fracture surgery along with how it relates to the general hip fracture population without dementia. To pinpoint interventions that may be used for reducing mortality in hip fracture patients with dementia further research into cause-specific mortality is warranted. Rather than merely focusing on allcause mortality in this patient population, this study aims to investigate the distribution and the risk of cause-specific mortality in hip fracture patients with dementia compared to the general hip fracture population.

\section{Methods}

After obtaining approval from the Swedish Ethical Review Authority (reference 2020-04161), all adult cases of hip fracture surgery, between January 1, 2008 and December 31,2017 , were retrieved from the prospectively collected Swedish National Quality Registry for Hip Fracture Patients, Rikshöft [11]. Patients whose hip fracture was managed nonoperatively, pathological fractures, and reoperations were excluded from the current study. The following data were retrieved from Rikshöft: date of hospital admission, age, sex, fracture type, American Society of Anesthesiologist (ASA) classification, surgical method, date of surgery, and hospital discharge date. The data from Rikshöft were crossreferenced with the Swedish National Board of Health and Welfare Patient and Cause of Death registers by matching patients' unique social security numbers, which allowed for the retrieval of data pertaining to time of death and comorbidities. All diagnoses registered for each social security number in the patient register, including dementia, were included as comorbidities. The comorbidity data were used to calculate the Charlson Comorbidity Index (CCI) [12]. The principles of the Declaration of Helsinki and STROBE guidelines were adhered to while conducting this study [13].

\section{Statistical analysis}

The cases were divided into two cohorts: patients with and without dementia. Demographics and clinical characteristics were compared between the cohorts; categorical variables are reported as percentages and continuous variables are reported as a mean and standard deviation (SD) or median and interquartile range (IQR). The statistical significance of differences between categorical variables was measured using Pearson's Chi-squared test. For continuous variables, the Student's $t$ test was used for normally distributed data, otherwise the Mann-Whitney $U$ test was applied. The primary outcome of interest was 30-day postoperative mortality.

A Poisson regression model was used to determine the association between dementia and 30-day all-cause postoperative mortality. The existence of hypertension, arrhythmias, peptic ulcer disease, and hemiplegia are reported in the cohort demographics but not adjusted for in the multivariate regression analyses. Hypertension and arrhythmia were not included since they are risk factors for other diagnoses, which were already adjusted for in the model, such as myocardial infarctions, congestive heart failure, and cerebrovascular events. Peptic ulcer disease was excluded as a result of its negligible association with mortality [14]. Adjusting for hemiplegia would risk overfitting the model since hemiplegia is often a result of a cerebrovascular event. The analysis was repeated to calculate the incidence rate ratio (IRR) for 30-day causespecific mortality for hip fracture patients with dementia compared to those without dementia. All analyses were performed while adjusting for age, sex, ASA classification, fracture type, type of surgery, and relevant comorbidities (prior myocardial infarctions, prior cerebrovascular events, peripheral vascular disease, chronic obstructive pulmonary disease, congestive heart failure, connective tissue diseases, diabetes mellitus, liver disease, chronic kidney disease, as well as local tumor and metastatic carcinoma). Multivariate imputation by chained equations was implemented to compensate for missing data in the covariates used in the regression model; logistic regression was used for binary variables, Bayesian polytomous regression for nominal variables, and a proportional odds model for ordinal variables.

Results are reported as IRRs with 95\% confidence intervals (CI). Statistical significance was defined as a two-sided $p$ value of less than 0.05. Analyses were performed using the statistical programming language R (R Foundation for Statistical Computing, Vienna, Austria) [15]. 


\section{Results}

A total of 134,915 cases met the study inclusion criteria. Patients with dementia tended to be older [mean (SD): 85 (11) vs 81 (7) years, $p<0.001]$ and females were more prevalent in both cohorts (dementia: $69.2 \%$, no dementia: $67.9 \%, p<0.001)$. Patients with dementia were less fit for surgery $(\mathrm{ASA} \geq 3: 71.2 \%$ vs $54.0 \%, p<0.001)$ and had more comorbidities (CCI $\geq 7: 28.5 \%$ vs $16.0 \%$, $p<0.001$ ) (Table 1). Hemiarthroplasty was undertaken to a larger extent in patients with dementia $(31.4 \%$ vs $24.2 \%$, $p<0.001)$ while total hip replacement was more common in patients without dementia $(8.7 \%$ vs $2.0 \%, p<0.001)$. There were no clinically significant differences in the distribution of fracture types (Table 1). Depicted in Table 2 are the prevalence of specific comorbidities within each cohort. Arrhythmia, congestive heart failure, cerebrovascular events, and peptic ulcer disease were more prevalent in patients with dementia, while peripheral vascular disease, chronic obstructive pulmonary disease, connective tissue disease, liver disease, diabetes mellitus, hemiplegia, chronic kidney disease, local tumor, and metastatic carcinoma were more common in patients without dementia (Table 2).

Crude 30-day all-cause postoperative mortality was twice as high in patients with dementia compared to those without dementia $(12.8 \%$ vs $6.2 \%, p<0.001)$. All specific causes of death were also more prevalent in patients with dementia (Table 3). In the Poisson regression analysis, the incidence of 30-day mortality was $67 \%$ higher in hip fracture patients with dementia [adj. IRR (95\% CI): 1.67 (1.60-1.75),
Table 1 Patient demographics and clinical characteristics in hip fracture cases undergoing surgery with and without dementia

\begin{tabular}{|c|c|c|c|}
\hline & $\begin{array}{l}\text { No dementia } \\
N=107,611\end{array}$ & $\begin{array}{l}\text { Dementia } \\
N=27,304\end{array}$ & $p$ value \\
\hline Age in years, mean [SD] & $81[11]$ & 85 [7] & $<0.001$ \\
\hline Sex, $n(\%)$ & & & $<0.001$ \\
\hline Female & $73,032(67.9)$ & $18,881(69.2)$ & \\
\hline Male & $34,566(32.1)$ & $8,422(30.8)$ & \\
\hline Missing & $13(0.0)$ & $1(0.0)$ & \\
\hline ASA classification, $n(\%)$ & & & $<0.001$ \\
\hline 1 & $6,359(5.9)$ & $297(1.1)$ & \\
\hline 2 & $41,227(38.3)$ & $7,037(25.8)$ & \\
\hline 3 & $50,120(46.6)$ & $16,737(61.3)$ & \\
\hline 4 & $7,866(7.3)$ & $2,668(9.8)$ & \\
\hline 5 & $95(0.1)$ & $40(0.1)$ & \\
\hline Missing & $1,944(1.8)$ & $525(1.9)$ & \\
\hline Charlson comorbidity index, $n(\%)$ & & & $<0.001$ \\
\hline$\leq 4$ & $57,030(53.0)$ & $2,581(9.5)$ & \\
\hline $5-6$ & $33,313(31.0)$ & $16,934(62.0)$ & \\
\hline$\geq 7$ & $17,268(16.0)$ & $7,789(28.5)$ & \\
\hline Fracture type, $n(\%)$ & & & $<0.001$ \\
\hline Non-displaced cervical (Garden 1-2) & $14,205(13.2)$ & $3,663(13.4)$ & \\
\hline Displaced cervical (Garden 3-4) & $39,789(37.0)$ & $10,383(38.0)$ & \\
\hline Basicervical & $3,541(3.3)$ & $939(3.4)$ & \\
\hline Pertrochanteric (two fragments) & $21,779(20.2)$ & $5,080(18.6)$ & \\
\hline Pertrochanteric (multiple fragments) & $19,206(17.8)$ & $5,287(19.4)$ & \\
\hline Subtrochanteric & $9,044(8.4)$ & $1,944(7.1)$ & \\
\hline Missing & $47(0.0)$ & $8(0.0)$ & \\
\hline Type of surgery, $n(\%)$ & & & $<0.001$ \\
\hline Pins or screws & $18,585(17.3)$ & $4,873(17.8)$ & \\
\hline Screws or pins with sideplate & $28,260(26.3)$ & $6,642(24.3)$ & \\
\hline Intramedullary rod & $25,353(23.6)$ & $6,639(24.3)$ & \\
\hline Hemiarthroplasty & $26,015(24.2)$ & $8,581(31.4)$ & \\
\hline Total hip replacement & $9,332(8.7)$ & $557(2.0)$ & \\
\hline Missing & $66(0.1)$ & $12(0.0)$ & \\
\hline
\end{tabular}

$S D$ standard deviation, ASA American Society of Anesthesiologists 
Table 2 Preoperative comorbidities in hip fracture cases undergoing surgery with and without dementia

\begin{tabular}{lcrr}
\hline & No dementia & Dementia & $p$ value \\
& $N=107,611$ & $N=27,304$ & \\
\hline Hypertension, $n(\%)$ & $41,294(38.4)$ & $10,462(38.3)$ & 0.87 \\
Arrhythmia, $n(\%)$ & $19,755(18.4)$ & $5,243(19.2)$ & $<0.001$ \\
Myocardial infarction, $n(\%)$ & $6,452(6.0)$ & $1,611(5.9)$ & 0.56 \\
Congestive heart failure, $n(\%)$ & $16,525(15.4)$ & $4,572(16.7)$ & $<0.001$ \\
Peripheral vascular disease, $n(\%)$ & $4,943(4.6)$ & $947(3.5)$ & $<0.001$ \\
Cerebrovascular event, $n(\%)$ & $17,477(16.2)$ & $5,905(21.6)$ & $<0.001$ \\
Chronic obstructive pulmonary disease, $n(\%)$ & $12,841(11.9)$ & $2,736(10.0)$ & $<0.001$ \\
Connective tissue disease, $n(\%)$ & $5,412(5.0)$ & $1,075(3.9)$ & $<0.001$ \\
Peptic ulcer disease, $n(\%)$ & $3,397(3.2)$ & $931(3.4)$ & 0.036 \\
Liver disease, $n$ (\%) & $1,185(1.1)$ & $185(0.7)$ & $<0.001$ \\
Diabetes mellitus, $n(\%)$ & $15,987(14.9)$ & $3,869(14.2)$ & 0.004 \\
Hemiplegia, $n(\%)$ & $2,441(2.3)$ & $470(1.7)$ & $<0.001$ \\
Chronic kidney disease, $n(\%)$ & $5,621(5.2)$ & $1,324(4.8)$ & 0.013 \\
Local tumor, $n(\%)$ & $11,893(11.1)$ & $2,667(9.8)$ & $<0.001$ \\
Metastatic carcinoma, $n(\%)$ & $2,595(2.4)$ & $367(1.3)$ & $<0.001$ \\
\hline
\end{tabular}

Table 3 Crude 30-day postoperative mortality in hip fracture cases undergoing surgery with and without dementia

\begin{tabular}{lrrr}
\hline & $\begin{array}{c}\text { No dementia } \\
N=107,611\end{array}$ & $\begin{array}{l}\text { Dementia } \\
N=27,304\end{array}$ & $p$ value \\
\hline $\begin{array}{l}\text { All-cause mortality } \\
\text { 30-day mortality, } n(\%)\end{array}$ & $6,640(6.2)$ & $3,490(12.8)$ & $<0.001$ \\
Cause-specific mortality & & & \\
Cardiovascular, $n(\%)$ & $2,895(2.7)$ & $1,287(4.7)$ & $<0.001$ \\
Respiratory, $n(\%)$ & $1,083(1.0)$ & $619(2.3)$ & $<0.001$ \\
Cerebrovascular, $n(\%)$ & $58(0.1)$ & $108(0.4)$ & $<0.001$ \\
Sepsis, $n(\%)$ & $118(0.1)$ & $74(0.3)$ & $<0.001$ \\
Multi-organ failure, $n(\%)$ & $2,413(2.2)$ & $1,176(4.3)$ & $<0.001$ \\
Unknown, $n(\%)$ & $73(0.1)$ & $226(0.8)$ & $<0.001$ \\
\hline
\end{tabular}

$p<0.001$ ] (Table 4). Patients with dementia had a 42\% [adj. IRR (95\% CI): $1.42(1.32-1.54), p<0.001$ ] and a 76\% [adj. IRR (95\% CI): 1.76 (1.57-1.99), $p<0.001]$ increased risk of death caused by postoperative cardiovascular and respiratory events, respectively. The risk of death due to sepsis was twice as high in patients with dementia [adj. IRR (95\% CI): 2.17 (1.47-3.22), $p<0.001]$. Patients with dementia also had a sevenfold increase in the risk of death resulting from cerebrovascular events [adj. IRR (95\% CI): 7.43 (4.99-11.07), $p<0.001$ ] (Table 4).

\section{Discussion}

To the authors' best knowledge, this is the first study investigating the risk of all-cause and cause-specific mortality in hip fracture patients with dementia compared to the
Table 4 Incidence rate ratio (IRR) for postoperative mortality after hip fracture surgery

\begin{tabular}{lll}
\hline Variable & $\begin{array}{l}\text { 30-day mortality IRR } \\
(95 \% \text { CI })\end{array}$ & $p$ value \\
\hline $\begin{array}{lll}\text { All-cause mortality } \\
\text { No dementia }\end{array}$ & Ref & \\
Dementia & $1.67(1.60-1.75)$ & $<0.001$ \\
Cause-specific mortality for patients with dementia* & \\
Cardiovascular & $1.42(1.32-1.54)$ & $<0.001$ \\
Respiratory & $1.76(1.57-1.99)$ & $<0.001$ \\
Cerebrovascular** & $7.43(4.99-11.07)$ & $<0.001$ \\
Sepsis & $2.17(1.47-3.22)$ & $<0.001$ \\
Multi-organ failure & $1.66(1.53-1.79)$ & $<0.001$ \\
Unknown*** & $11.2(8.06-15.58)$ & $<0.001$ \\
\hline
\end{tabular}

Poisson regression model with robust standard errors. Multiple imputation with chained equations was used to manage missing values. The model is adjusted for age, sex, American Society of Anesthesiologists (ASA) classification, fracture type, type of surgery, prior myocardial infarctions, prior cerebrovascular events, peripheral vascular disease, chronic obstructive pulmonary disease, congestive heart failure, connective tissue diseases, diabetes mellitus, liver disease, chronic kidney disease, as well as local tumor and metastatic carcinoma

*Reference group is patients without dementia

** Not adjusted for ASA classification and liver disease due to their inclusion resulting in a matrix that is computationally singular

*** Not adjusted for ASA classification due to its inclusion resulting in a matrix that is computationally singular

general hip fracture population. In this large nationwide cohort study, patients with dementia had twice the risk of postoperative all-cause mortality compared to patients without dementia. After adjusting for differences between the 
cohorts, there was a substantially increased risk of death due to cardiovascular, pulmonary, cerebrovascular, and septic events in patients with dementia.

The relationship between dementia and postoperative mortality has long been known by orthopedic surgeons [1-4]. In a meta-analysis by Rao et al. from 2016, comparing hospital outcomes in all patients with and without dementia, the authors found that the relative risk of allcause mortality was $70 \%$ higher in patients with dementia. This corresponded to an overall mortality rate of $15.3 \%$ in dementia patients compared to $8.7 \%$ in non-dementia cases. Patients with dementia also had higher readmission rates and longer lengths of stay in the hospital. However, they also underwent fewer life-saving interventions and procedures [16]. Similar results were found in our study where the relative risk of all-cause mortality was increased by $67 \%$ in patients with dementia. The crude mortality rate was $12.8 \%$ in patients with dementia compared to $6.2 \%$ in patients without dementia. To be able to pinpoint which interventions aimed at reducing mortality are worth investigating, research into the specific causes of mortality is required. Previous studies have primarily been concerned with the distribution of causes of mortality in patients who died with dementia, as opposed to the risk for such events compared to patients without dementia $[17,18]$.

Dementia has often been referred to as the cause of mortality in previous, often orthopedic, studies [18-22]. The current study demonstrates that this line of reasoning is misleading. Patients with dementia have a higher risk of postoperative death, but they still die from the failure of a specific organ or multiple organ systems. Labeling the cause of death as dementia obscures this fact and lulls physicians into a false sense of security by promoting the reasoning 'there was nothing more we could have done'. Dementia is a risk factor that predicts mortality, not the end cause [2-4].

Hip fracture patients with dementia should be considered a distinct patient population in orthopedic surgery in need of particular care $[2,16]$. In light of this, the use of risk stratification tools in the clinical setting may be of interest. Several tools have been investigated in the context of the general hip fracture population, such as the Nottingham Hip Fracture Score, CCI, and Physiological and Operative Severity Score for the enUmeration of Mortality and Morbidity (POSSUM) [12, 23-25]. Risk stratification may aid in attaining better resource allocation, patient prioritization, preoperative optimization, and monitoring. More significantly, it can assist orthopedic surgeons and their multidisciplinary teams in identifying patients who might gain from specialized postoperative care.

Old age, frailty, polypharmacy, comorbidities, and dementia are well-known risk factors for delirium in hospital patients, particularly postoperative delirium [26-29]. All these factors are common in hip fracture patients $[7,8$,
19, 30-34], and even more so in those with dementia, resulting in delirium being a familiar complication on orthopedic wards. Postoperative delirium episodes have been strongly linked to an increased mortality risk, especially in the emergency setting [28]. As it stands, multiple guidelines have been proposed recently to reduce the risk of delirium in geriatric patients, which may warrant further examination and further adjustment for patients with dementia [35-37]. Reducing the risk of delirium could potentially decrease the risk of many of the specific causes of death after hip fracture surgery, particularly in the more vulnerable population of patients with dementia.

Due to the retrospective nature of the current study, the authors cannot make any certain claims about the increased risk of respiratory death detected. The high proportion of respiratory deaths has previously been noted in the general dementia population which is consistent with the current findings $[17,18]$. This is an important finding since postoperative measures to decrease the risk of respiratory complications, which have a higher incidence postoperatively, could be emphasized more in this patient population. Further investigations are required to determine which perioperative interventions are needed to reduce each specific postoperative mortality risk in hip fracture patients with dementia.

This study is based on 10 consecutive years of data from the Swedish National Quality Registry for Hip Fracture Patients, which is known for having a high case coverage between 80 and $90 \%$ [38]. As a result of the universal healthcare system in Sweden, patient management is also relatively uniform across all orthopedic departments. Furthermore, the nature of this system removes many of the socioeconomic barriers that might result in some patients going undiagnosed and undertreated for serious health conditions. However, several limitations to the current study are worth mentioning. Due to the retrospective nature of the study, surgical experience, postoperative pain, and functional outcome could not be included in the analysis. In addition, while dementia has been treated as a single monolithic disease in the current study, the reality is that dementia is the result of several different conditions with varying pathophysiology and mortality rates $[17,18]$. The authors recognize that it is possible that a distinction between these different conditions could result in different mortality rates.

\section{Conclusion}

This study demonstrates that hip fracture patients with dementia have a higher risk of death in the first 30 days postoperatively, with a substantially higher risk of cardiovascular, respiratory, and cerebrovascular mortality specifically. These findings could be used as the basis for better risk assessments in this patient population, and aid improved 
perioperative monitoring and care optimization, to achieve better overall outcomes after hip fracture surgery.

Author contributions II: study design, analysis and interpretation of the data, drafting and revision of manuscript. AMI: study design, data collection, analysis and interpretation of the data, drafting and revision of manuscript. MPF: data collection, analysis and interpretation of the data, drafting and revision of manuscript. RA: analysis and interpretation of the data, drafting and revision of manuscript. YC: analysis and interpretation of the data, drafting and revision of manuscript. TB: interpretation of the data, drafting and revision of manuscript. SM: study design, data collection, analysis and interpretation of the data, drafting and revision of manuscript. All the authors have approved the submitted version of the manuscript.

Funding Open access funding provided by Örebro University.

Code availability All codes are available upon reasonable request.

\section{Compliance with ethical standards}

Conflict of interest All the authors certify that they have no affiliations with or involvement in any organization or entity with any financial interest or non-financial interest in the subject matter or materials discussed in this manuscript.

Ethics approval Swedish Ethical Review Authority (reference 202004161).

Availability of data and material All data are available upon reasonable request from the editorial board.

Open Access This article is licensed under a Creative Commons Attribution 4.0 International License, which permits use, sharing, adaptation, distribution and reproduction in any medium or format, as long as you give appropriate credit to the original author(s) and the source, provide a link to the Creative Commons licence, and indicate if changes were made. The images or other third party material in this article are included in the article's Creative Commons licence, unless indicated otherwise in a credit line to the material. If material is not included in the article's Creative Commons licence and your intended use is not permitted by statutory regulation or exceeds the permitted use, you will need to obtain permission directly from the copyright holder. To view a copy of this licence, visit http://creativecommons.org/licenses/by/4.0/.

\section{References}

1. Bai J, Zhang P, Liang X, Wu Z, Wang J, Liang Y. Association between dementia and mortality in the elderly patients undergoing hip fracture surgery: a meta-analysis. J Orthop Surg Res. 2018. https://doi.org/10.1186/s13018-018-0988-6.

2. Chiu H-C, Chen C-M, Su T-Y, Chen C-H, Hsieh H-M, Hsieh C-P, Shen D-L. Dementia predicted one-year mortality for patients with first hip fracture. Bone Jt J. 2018;100:1220-6.

3. Dutkiewicz R, Zetterberg H, Andreasson U, Blennow K, Nellgård B. Dementia and CSF-biomarkers for Alzheimer's disease predict mortality after acute hip fracture. Acta Anaesthesiol Scand. 2020;64:93-103.
4. Clague JE, Craddock E, Andrew G, Horan MA, Pendleton N. Predictors of outcome following hip fracture. Admission time predicts length of stay and in-hospital mortality. Injury. 2002;33:1-6.

5. Kanis JA, Odén A, McCloskey EV, Johansson H, Wahl DA, Cooper C, IOF Working Group on Epidemiology and Quality of Life. A systematic review of hip fracture incidence and probability of fracture worldwide. Osteoporos Int. 2012;23:2239-56.

6. Bloom BS, Banta HD, Gross PF, Peña-Mohr J, Sisk JE, Stocking B. The Swedish council on technology assessment in health care. Int J Technol Assess Health Care. 1989;5:154-8.

7. Forssten MP, Mohammad Ismail A, Sjolin G, Ahl R, Wretenberg $\mathrm{P}$, Borg T, Mohseni S. The association between the revised cardiac risk index and short-term mortality after hip fracture surgery. Eur J Trauma Emerg Surg. 2020. https://doi.org/10.1007/s00068-02001488-w.

8. Mohammad Ismail A, Borg T, Sjolin G, Pourlotfi A, Holm S, Cao Y, Wretenberg P, Ahl R, Mohseni S. $\beta$-adrenergic blockade is associated with a reduced risk of 90-day mortality after surgery for hip fractures. Trauma Surg Acute Care Open. 2020;5:e00533.

9. Nationella riktlinjer för vård och omsorg vid demenssjukdom. In: Socialstyrelsen. https://www.socialstyrelsen.se/regler-och-riktl injer/nationella-riktlinjer/publicerade-riktlinjer/demens/2018-3-1. pdf. Accessed 11 Sep 2020

10. Tomaskova H, Kuhnova J, Cimler R, Dolezal O, Kuca K. Prediction of population with Alzheimer's disease in the European Union using a system dynamics model. Neuropsychiatr Dis Treat. 2016;12:1589-98.

11. Rikshoft. Swedish National Registry of hip fracture patient care. https://www.xn--rikshft-e1a.se/om-oss. Accessed December 25 2020.

12. Charlson ME, Pompei P, Ales KL, MacKenzie CR. A new method of classifying prognostic comorbidity in longitudinal studies: development and validation. J Chronic Dis. 1987;40:373-83.

13. WMA - The World Medical Association-WMA Declaration of Helsinki - Ethical Principles for Medical Research Involving Human Subjects.

14. Peptic ulcer | United States| PDF | PPT| Case Reports | Symptoms I Treatment. https://www.omicsonline.org/united-states/pepti c-ulcer-peer-reviewed-pdf-ppt-articles/. Accessed 4 Oct 2020

15. R Development Core Team. R: a language and environment for statistical computing. Vienna: R Foundation for Statistical Computing; 2008.

16. Rao A, Suliman A, Vuik S, Aylin P, Darzi A. Outcomes of dementia: systematic review and meta-analysis of hospital administrative database studies. Arch Gerontol Geriatr. 2016;66:198-204.

17. Brunnström HR, Englund EM. Cause of death in patients with dementia disorders. Eur J Neurol. 2009;16:488-92.

18. Garcia-Ptacek S, Kåreholt I, Cermakova P, Rizzuto D, Religa D, Eriksdotter M. Causes of death according to death certificates in individuals with dementia: a cohort from the Swedish Dementia Registry. J Am Geriatr Soc. 2016;64:e137-42.

19. Panula J, Pihlajamäki H, Mattila VM, Jaatinen P, Vahlberg T, Aarnio P, Kivelä S-L. Mortality and cause of death in hip fracture patients aged 65 or older: a population-based study. BMC Musculoskelet Disord. 2011;12:105.

20. Berggren M, Stenvall M, Englund U, Olofsson B, Gustafson Y. Co-morbidities, complications and causes of death among people with femoral neck fracture-a three-year follow-up study. BMC Geriatr. 2016. https://doi.org/10.1186/s12877-016-0291-5.

21. Mitchell SL, Teno JM, Kiely DK, Shaffer ML, Jones RN, Prigerson HG, Volicer L, Givens JL, Hamel MB. The clinical course of advanced dementia. N Engl J Med. 2009;361:1529-38.

22. Falk N, Cole A, Meredith TJ. Evaluation of suspected dementia. AFP. 2018;97:398-405.

23. Maxwell MJ, Moran CG, Moppett IK. Development and validation of a preoperative scoring system to predict 30 day 
mortality in patients undergoing hip fracture surgery. Br J Anaesth. 2008;101:511-7.

24. Copeland GP, Jones D, Walters M. POSSUM: a scoring system for surgical audit. Br J Surg. 1991;78:355-60.

25. Prytherch DR, Whiteley MS, Higgins B, Weaver PC, Prout WG, Powell SJ. POSSUM and Portsmouth POSSUM for predicting mortality. Physiological and operative severity score for the enumeration of mortality and morbidity. Br J Surg. 1998;85:1217-20.

26. Fong TG, Davis D, Growdon ME, Albuquerque A, Inouye SK. The interface of delirium and dementia in older persons. Lancet Neurol. 2015;14:823-32.

27. Ford AH. Preventing delirium in dementia: managing risk factors. Maturitas. 2016;92:35-40.

28. Saljuqi AT, Hanna K, Asmar S, Tang A, Zeeshan M, Gries L, Ditillo M, Kulvatunyou N, Castanon L, Joseph B. Prospective evaluation of delirium in geriatric patients undergoing emergency general surgery. J Am Coll Surg. 2020;230:758-65.

29. Kang SY, Seo SW, Kim JY. Comprehensive risk factor evaluation of postoperative delirium following major surgery: clinical data warehouse analysis. Neurol Sci. 2019;40:793-800.

30. Gundel O, Thygesen LC, Gögenur I, Ekeloef S. Postoperative mortality after a hip fracture over a 15 -year period in Denmark: a national register study. Acta Orthop. 2020;91:58-62.

31. Choi HG, Lee YB, Rhyu SH, Kwon BC, Lee JK. Mortality and cause of death postoperatively in patients with a hip fracture: a national cohort longitudinal follow-up study. Bone Jt J. 2018;100:436-42.
32. Cher EWL, Allen JC, Howe TS, Koh JSB. Comorbidity as the dominant predictor of mortality after hip fracture surgeries. Osteoporos Int. 2019;30:2477-83.

33. Schnell S, Friedman SM, Mendelson DA, Bingham KW, Kates SL. The 1-year mortality of patients treated in a hip fracture program for elders. Geriatr Orthop Surg Rehabil. 2010;1:6-14.

34. Arsrapport R. Rikshoft. Sweden: Lund; 2018.

35. Hughes CG, Boncyk CS, Culley DJ, Fleisher LA, Leung JM, McDonagh DL, Gan TJ, McEvoy MD, Miller TE. American Society for enhanced recovery and perioperative quality initiative joint consensus statement on postoperative delirium prevention. Anesth Analg. 2020;130:1572-90.

36. Berger M, Schenning KJ, Brown $\mathrm{CH}$, et al. Best practices for postoperative brain health: recommendations from the fifth international perioperative neurotoxicity working group. Anesth Analg. 2018;127:1406-13.

37. American Geriatrics Society Expert Panel on Postoperative Delirium in Older Adults. Postoperative delirium in older adults: best practice statement from the American Geriatrics Society. J Am Coll Surg. 2015;220:136-148.e1.

38. Meyer AC, Hedström M, Modig K. The Swedish hip fracture register and national patient register were valuable for research on hip fractures: comparison of two registers. J Clin Epidemiol. 2020;125:91-9. 